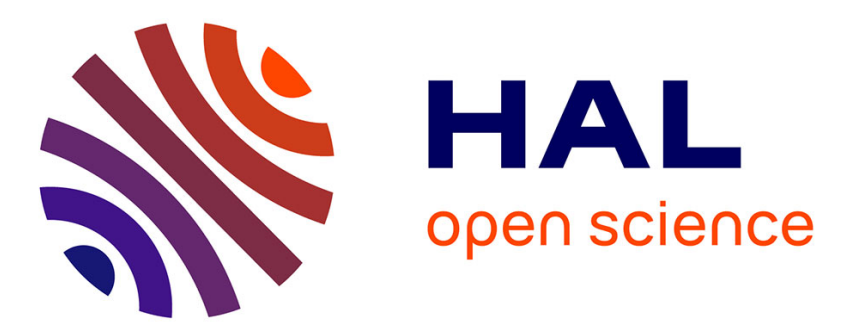

\title{
Application of wave distribution function methods to an ELF hiss event at high latitudes
}

\author{
Ondřej Santolik, Michel Parrot
}

\section{To cite this version:}

Ondřej Santolik, Michel Parrot. Application of wave distribution function methods to an ELF hiss event at high latitudes. Journal of Geophysical Research Space Physics, 2000, 105, pp.18885-18894. insu-03234738

\section{HAL Id: insu-03234738 \\ https://hal-insu.archives-ouvertes.fr/insu-03234738}

Submitted on 25 May 2021

HAL is a multi-disciplinary open access archive for the deposit and dissemination of scientific research documents, whether they are published or not. The documents may come from teaching and research institutions in France or abroad, or from public or private research centers.
L'archive ouverte pluridisciplinaire $\mathbf{H A L}$, est destinée au dépôt et à la diffusion de documents scientifiques de niveau recherche, publiés ou non, émanant des établissements d'enseignement et de recherche français ou étrangers, des laboratoires publics ou privés. 


\title{
Application of wave distribution function methods to an ELF hiss event at high latitudes
}

\author{
Ondřej Santolík ${ }^{1}$ \\ Faculty of Mathematics and Physics, Charles University, Prague, Czech Republic \\ Michel Parrot \\ Laboratoire de Physique et Chimie de l'Environnement, Centre National de la Recherche Scientıfique, \\ Orléans, France
}

\begin{abstract}
Multicomponent waveform data are used to calculate wave polarization and propagation characteristics, with both simple plane wave methods and more general wave distribution function (WDF) techniques. The WDF techniques allow us to estimate a continuous distribution of wave energy with respect to the wave vector directions. We describe several newly developed analysis methods, and we compare their results with existing techniques. This comparison allows us to reach a common interpretation of propagation properties and to exclude possible artifacts. The methods are applied to an electromagnetic ELF hiss event observed by the low-altitude satellite Freja. A part of the emission can be described by simple plane wave methods. WDF techniques are, however, needed to fully characterize the wave propagation when the components of the wave magnetic field vector are not mutually coherent and the polarization degree is low. In this case we observe large peaks on the WDF shape or simultaneous propagation of downgoing and reflected upgoing waves. The WDF techniques also confirm that experimental polarization properties are consistent with their predictions by the cold plasma theory.
\end{abstract}

\section{Introduction}

Various techniques can be used to estimate the wave polarization and propagation characteristics. The majority of them supposes the presence of a single plane wave in a single wave mode. The wave-normal direction can be estimated by different methods [e.g., Means, 1972; McPherron et al., 1972; Samson and Olson, 1980] using always the fact that the wave magnetic field is perpendicular to the wave vector. The plane wave analysis is a very useful tool because the results are simple and can be easily represented in time-frequency plots [Santolik and Parrot, 1999]. However, in some wave emissions (e.g., ELF hiss) the wave field is rather complex, and the supposition of the presence of a single plane wave becomes invalid. In such a case, a continuous distribution of wave vector directions can be described by the wave distribution function (WDF), as first proposed by Storey and Lefeuvre [1974, 1979, 1980]. The WDF defines the distribution of the wave energy density with respect to the frequency and wave vector direction. Lefeuvre and Delannoy [1979] developed a technique of the WDF estimation based on the maximum entropy method. With this method, numerous cases of ELF hiss have been analyzed, at high altitudes [Lefeuvre and Helliwell, 1985; Parrot and Lefeuvre, 1986; Storey et al.,

\footnotetext{
${ }^{1}$ Also at Institute of Atmospheric Physics, Academy of Sciences of the Czech Republic, Prague.
}

Copyright 2000 by the American Geophysical Union.

Paper number 2000JA900029.

0148-0227/00/2000JA900029\$09.00
1991], and also at low altitudes [Lefeuvre et al., 1992; Santolik and Parrot, 1998].

In the present paper, we use both plane wave estimations and different methods of WDF analysis to process an ELF hiss event recorded by the low-altitude Freja satellite (for details about the mission see Space Science Reviews, 70(3/4), 1994). The F4 wave experiment [Holback et al., 1994] measures waveforms of three orthogonal magnetic components and one electric component received by a spinning antenna. We transform the magnetic components into the coordinate system connected with the Earth's magnetic field $\mathbf{B}_{0}$ : The $z$ axis is parallel to $\mathbf{B}_{0}$, and the $x$ axis points toward the direction of decreasing magnetic latitude in the magnetic meridian plane (Figure 1). The local direction of the DC field is provided by the $\mathrm{F} 2$ fluxgate magnetometer [FREJA magnetic field experiment team, 1994]. The transformed magnetic waveforms as well as the electric waveform are then subjected to a spectral analysis. We estimate a complex spectrum $b_{i}(f)$ of the $i$ th component at a frequency $f$, and the spectral matrix is then defined as $S_{i j}(f)=\left\langle b_{i}(f) b_{j}^{*}(f)\right\rangle$, where \langle\rangle means time average. The spectral matrices contain information about the power-spectral density, relative phases, and mutual coherency of the field components. These matrices are used as the input data for both plane wave and WDF analysis methods. The plane wave methods provide the first approximation of the wave propagation characteristics. They also allow an overall view over the whole time and frequency interval under study. In selected points of the time-frequency plane, we estimate the WDF shape with four independent methods. We use the original technique of Lefeuvre and Delannoy [1979], as well as three newly developed procedures. The comparison of results allows us to estimate method-dependent uncertainties of the obtained WDF shape and to facilitate its interpretation. 


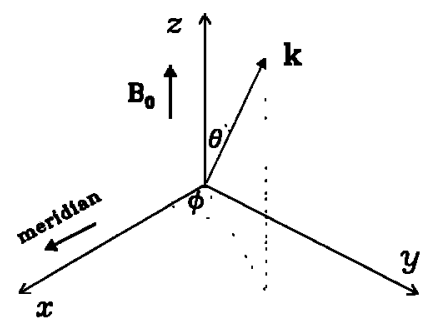

Figure 1. Spherical coordinates used to describe the wave vector direction.

The main aim of the present technical paper is thus the comparison of different WDF methods and the examination of their consistence with the plane wave analysis in the time-frequency plane. In section 2 we will present a typical case of ELF hiss with poleward reflection and its plane-wave analysis. Then, the WDF approach will be described and discussed. Finally, conclusions will be given. The appendix contains a description of the WDF analysis techniques.

\section{Plane Wave Analysis}

On April 8, 1995, between 1548 and 1558 UT, Freja observed a very intense emission of ELF hiss. Intense waves below $f_{\mathrm{H}}+$ were associated with this emission [Santolik and Parrot, 1998]. In this time interval the satellite operated at altitudes between 900 and $1300 \mathrm{~km}$ and moved from $50^{\circ}$ to $74^{\circ}$ of corrected geomagnetic latitude (CGLat). It was located in the morning sector, from 0545 to 0930 magnetic local time (MLT). Plates 1a and 1b show power spectrograms of the magnetic $B_{x}$ component and of the electric component. While the magnetic component is expressed in the coordinate system illustrated in Figure 1, the electric component is measured by the spinning antenna. The electric spectrogram therefore contains artificial intensity enhancements with a period of $\sim 12 \mathrm{~s}$, which result from the interference between the 6-s spin period and the 8-s cycle of waveform snapshots. The periodically repeated triplets of vertical red lines are also due to instrumental effects. Around 1556:15 UT a short data gap (in dark blue) is present in both spectrograms. Regardless of these few petty artifacts we can clearly see the electromagnetic ELF hiss emission with a lower cutoff just below the local proton gyrofrequency $\left(f_{\mathrm{H}^{+}}\right)$. The cutoff can be detected in all field components, but it is better expressed in the $B_{z}$ data (not shown). Before 1555 UT the hiss has no stable and clearly defined upper frequency limitation. After this time, the emission is limited in a band below $\sim 750$ Hz. Besides ELF hiss, two other intense emissions are present in the spectrograms. Before 1555 UT we detect waves below $f_{\mathrm{H}^{+}}$[Santolik and Parrot, 1998]. Around 1555:30 an intense emission of broadband low-frequency electromagnetic noise is observed. Results of recognition of upgoing and downgoing waves are presented in Plate 1c. We use a method based on the data of the spinning electric antenna and on the simultaneous measurement of the full vector of the wave magnetic field [Santolik and Parrot, 1999]. This procedure provides the phase between perpendicular magnetic and electric field components. A phase of $0^{\circ}$ corresponds to a positive $z$ component of the wave vector, i.e. downgoing waves in the northern high-latitude region (see Figure 1), and oppositely, a phase of $180^{\circ}$ means a negative $z$ component, i.e., upgoing waves. We observe that the majority of observed emissions are downgoing, with an important exception of the band-limited part of hiss after $1555 \mathrm{UT}$, which clearly propagates upward.

The ellipticity of the magnetic field polarization could be calculated from the eigenvector associated with the maximum eigenvalue of the magnetic spectral matrix [Samson and Olson, 1980]. The results (not shown) indicate that nearly whole hiss emission is elliptically or nearly circularly polarized. However, a narrow band just below the local $f_{\mathrm{H}^{+}}$, observed before 1555:30 UT, has a low ellipticity. A very low ellipticity is also found in the low-frequency electromagnetic noise. This is consistent with previous results [Santolik and Parrot, 1999]. As concerns the sense of the elliptic polarization, the ELF hiss propagates in the right-hand polarized mode above $f_{\mathrm{H}^{+}}$[Gurnett and Burns, 1968]. This is the only mode predicted at these frequencies by the cold plasma theory [Stix, 1992]. In the case under study, the analysis of the sense of wave polarization with respect to $\mathbf{B}_{0}$ also gives right-handed polarization for all elliptically polarized emissions (not shown).

Using the plane wave approximation, the wave vector direction is fully determined by the polar angle $\theta$ and the azimuthal angle $\phi$ (see Figure 1). These angles have been estimated by the method of Means [1972]. The procedure is based on the magnetic field data only and therefore does not take into account the difference between upward and downward propagation. For upgoing waves the method gives the $\theta$ and $\phi$ values which correspond to the antiparallel downward propagation. Thus we must use previous recognition of downgoing and upgoing waves (Plate 1c) to eliminate this ambiguity and to correctly interpret obtained $\theta$ and $\phi$ values. In the case of the ELF hiss emission we observe a complex evolution of the wave vector direction across the time-frequency plane, which is similar to some cases of "type A" emissions described by Santolik and Parrot [1999]. However, in the present paper the data much more clearly indicate poleward reflection of originally downgoing hiss. For the sake of simplicity, we show only the results in a selected frequency interval as a three-dimensional plot in magnetic coordinates. In Figure 2, a part of the satellite orbit between 1549 UT and 1557 UT is given by a thick line. The magnetic latitude is represented by parallels, from CGLat of $50^{\circ}$ up to the pole. The magnetic meridians are plotted from 0400 to 1000 MLT. The parallels and meridians are placed on the Earth's surface, and the satellite orbit is plotted taking into account its real altitude. Figure 2 shows an orthogonal projection onto a plane declined by $45^{\circ}$ above the equator at $0212 \mathrm{MLT}$. At each round minute of UT a magnetic field line passing through the satellite position is plotted by a dotted line. Each line begins at the Earth's surface and goes up to $2000 \mathrm{~km}$ of altitude. Finally, the wave propagation directions are given by thin solid lines beginning at the satellite orbit and ended by cone-shaped arrowheads. When the vertex of a cone rests on the tip of the direction line, the waves propagate downward, and oppositely, a cone posed on its base means upward propagation. The presented directions have been obtained by 1 -min averages of the propagation parameters in the frequency range between 620 and $640 \mathrm{~Hz}$. The length of each direction line represents the logarithm of wave intensity given by the sum of spectral densities of the magnetic components. The zero length corresponds to intensity of $10^{-7} \mathrm{nT}^{2} / \mathrm{Hz}$, and the maximum length of $2000 \mathrm{~km}$ would be plotted for $10^{-4}$ $\mathrm{nT}^{2} / \mathrm{Hz}$. The projection in Figure 2 shows that waves at latitudes below $60^{\circ}$ propagate downward, and their wave vector direction is declined toward the equator. At a latitude of $\sim 62^{\circ}$ the wave vectors turn poleward, and above $70^{\circ}$, upward directed 
(a)
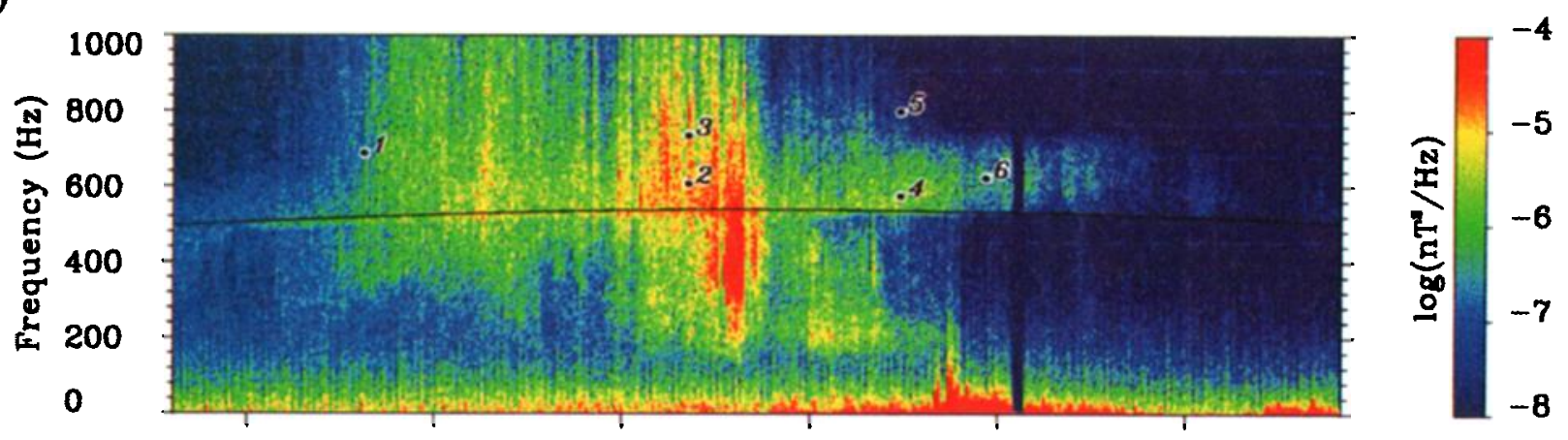

(b)
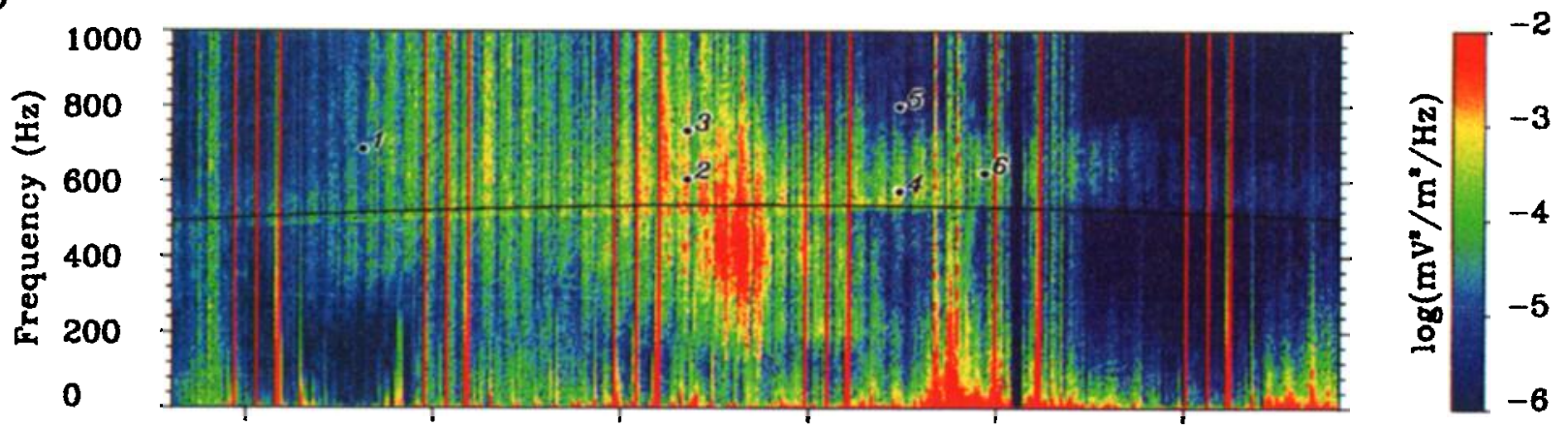

(c)
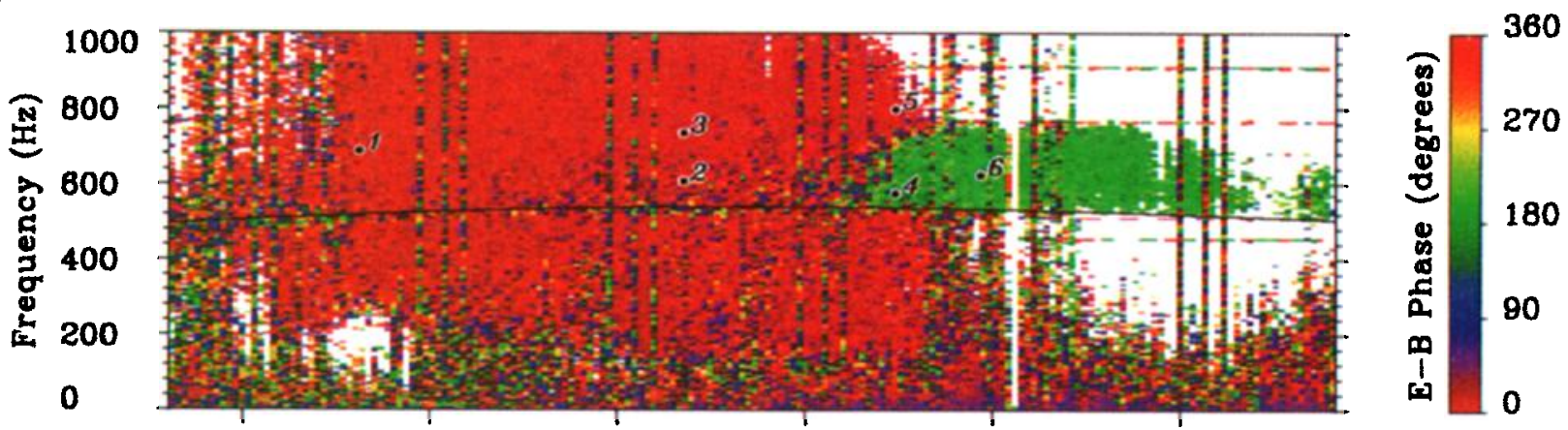

(d)

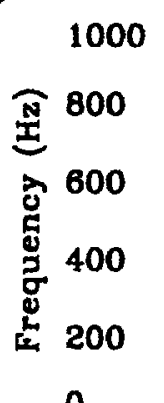

UT

MLT

CGLat

Alt
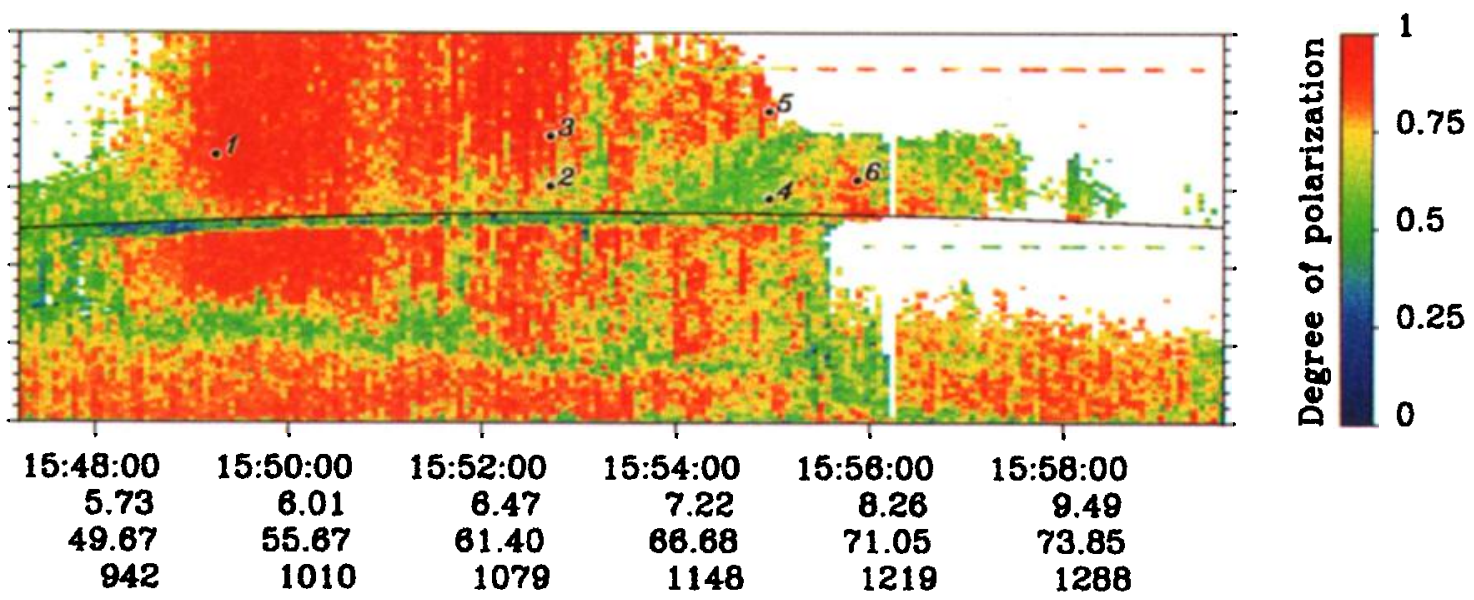

Plate 1. Results of the plane wave analysis (see text) on April 8, 1995. (a) Power spectrogram of magnetic $B_{x}$ component. (b) Power spectrogram of the electric field measured by the spinning antenna. (c) Phase between the perpendicular magnetic and electric components; red means downgoing waves, and green means upgoing waves. (d) Degree of polarization. White regions in Plates 1c and 1d correspond to low signal intensity. In Plates 1c-1d, black line indicates the proton gyrofrequency obtained from the local measurement of the dc magnetic field. Six points denoted by numbers 1-6 are selected for the WDF analysis. 


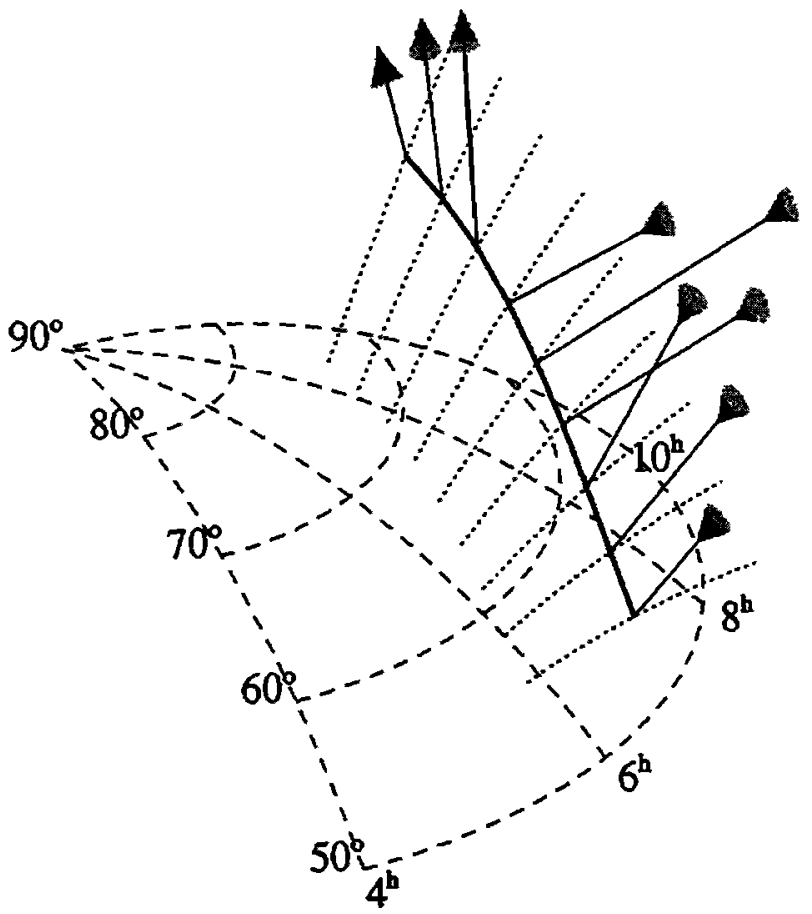

Figure 2. An orthogonal projection of the satellite orbit between 1549 UT and 1557 UT (thick solid line); the magnetic meridians between 0400 and 1000 MLT and the magnetic parallels for CGLat between $50^{\circ}$ and $90^{\circ}$ (dashed lines); the magnetic field lines between 0 and $2000 \mathrm{~km}$ of altitude, calculated for the satellite positions at each round minute of UT (dotted lines); and the average wave vector directions at these points (thin solid lines ended by shaded cones).

waves continue the poleward propagation at highly oblique $\theta$.

Plate 1d gives important information about the validity of the plane wave approximation. The degree of polarization is estimated by the method of Samson and Olson [1980]. If its value is equal to 1 , the magnetic field components are mutually coherent. This is consistent with the presence of a single plane wave in a single wave mode. Lower values of the polarization degree indicate the invalidity of the plane wave hypothesis. A very sharp decrease of the polarization degree in the narrow band just below the local $f_{\mathrm{H}^{+}}$could be attributed to complex wave mode and/or propagation structures in this particular interval. The data further show that the plane wave approximation is appropriate in some part of the hiss emission above $f_{\mathrm{H}}$, especially at lower latitudes and higher frequencies. Near the beginning of the upgoing part of the emission, after 1554 UT, the polarization degree decreases in a band of several hundreds of hertz above $f_{\mathrm{H}+}$. A decrease is also observed in a similar frequency band above $f_{\mathrm{H}}+$ for the nearly field-aligned hiss between 1551 and 1554 UT. For these waves we can expect the presence of more complex properties of the wave propagation than supposed by the simple plane wave approximation, and the WDF techniques should be used.

\section{WDF Analysis}

We will present results of the WDF analysis in six different points inside the ELF hiss spectrogram (noted by numbers 1-6 in Plates 1a-1c). They have been selected to show typical WDFs for all principal regimes of wave propagation: Point 1 is situated at lower latitudes, in the highly polarized initial part, where the plane wave analysis has found downgoing and equatorward propagating hiss at low $\theta$. Points 2 and 3 are located in the most intense part of downgoing and field-aligned hiss, in the frequency bands with lower or higher polarization, respectively. Points 4 and 5 are in the weaker part of the emission at higher latitudes. Here, previous analysis has shown upward propagating waves with a low polarization degree at lower frequencies (point 4) and downgoing waves at higher frequencies (point 5). In both cases the propagation is poleward and at high $\theta$. Finally, point 6 represents the high-latitude band-limited part of the hiss emission, which is, according to the plane wave analysis, purely upgoing and poleward propagating.

The WDF analysis requires theoretical prediction of the wave spectral matrices (see appendix for details). These calculations are based on a model of the plasma medium. In all cases we have used the cold plasma approximation which is well justified for description of the ELF wave propagation at low altitudes. We have further supposed a collisionless singly ionized oxygenhydrogen plasma. The presence of helium cannot be excluded in the ionospheric region under study, but it has probably low importance for the wave propagation above the local $f_{\mathrm{H}^{+}}$. The medium model is then fully described by its plasma frequency, by the gyrofrequencies, and by relative fractions of both ions. We obtain the plasma frequency from the measurements of the plasma density by a Langmuir probe. The gyrofrequencies are directly calculated from data provided by a fluxgate magnetometer. Finally, the ion composition is estimated using the value of the lower hybrid frequency identified with the lower cutoff of electrostatic waves at several kilohertz. It can be alternatively obtained from the position of the sharp lower cutoff of downgoing ELF hiss. For the six example points the values of the plasma frequency are always around $600 \mathrm{kHz}$, and the hydrogen fraction is $\sim 5 \%$. Rough upper estimates of the experimental errors are on the order of $100 \mathrm{kHz}$ for the plasma frequency and of several percent for the hydrogen fraction. We have verified that such uncertainties do not substantially influence the results.

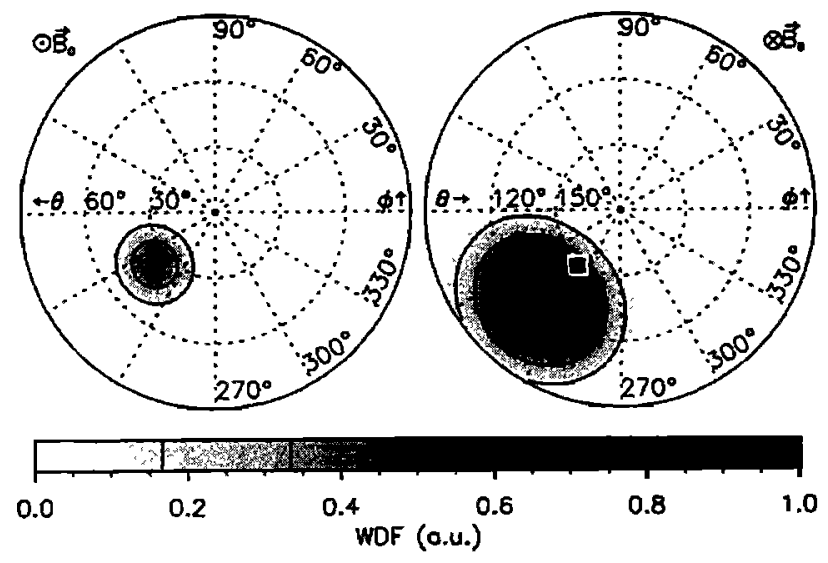

Figure 3. Example of graphical representation of the WDF analysis results. (left) Downgoing waves, (right) upgoing waves. Both polar diagrams show the $\theta$ - $\phi$ distribution of the wave energy density at a given frequency. The square sign represents the result of the plane wave analysis. 


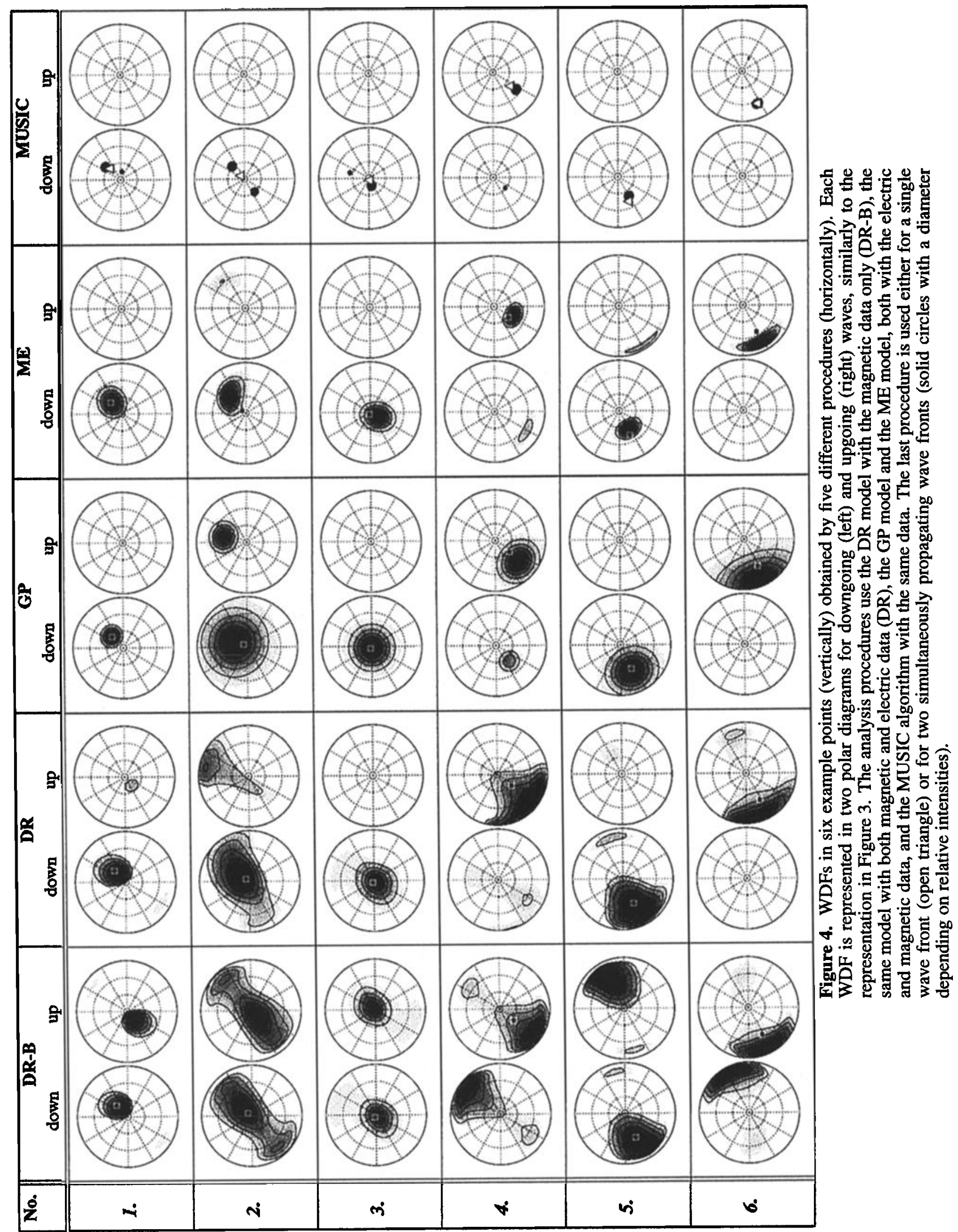


Using theoretically predicted spectral matrices, we have searched for the WDFs which are most consistent with a given experimental spectral matrix. We have used least squares techniques with three independent parametric models: the maximum entropy (ME) model, the model of Gaussian peaks (GP), and the model of discrete regions (DR). Additional independent information is provided by the multiple signal classification (MUSIC) algorithm, which is based on the eigenanalysis of the spectral matrix. This algorithm has been implemented for the WDF analysis (L. R. O. Storey, personal communication, 1998), and it provides the normal directions and intensities for a predefined number of incident wave fronts (for details see the appendix). Figure 3 shows an example of resulting WDF obtained by the GP model in point 4 . It is represented by two polar $\theta-\phi$ diagrams, for downgoing waves on the left, and for upgoing waves on the right. The WDF in arbitrary units is represented using the grey scale shown below and by five isocontours. To compare the results with the plane wave analysis, we overplot a square sign in the direction given by the method of Means [1972] and by the simultaneous recognition of upgoing and downgoing waves. Similar but condensed diagrams for all the six example points inside the hiss emission are shown in a comprehensive Figure 4. The first WDF estimate is based on the DR model and uses only the data from the three magnetic antennae. The second one uses the same WDF model but involves also the electric antenna. Obviously, these results are not the same although they use the same WDF model: The magnetic field data do not contain information about the downgoing and upgoing waves and the first procedure gives a symmetric WDF shape in both polar diagrams. With both magnetic and electric field data we are able to recognize whether the waves propagate downward or upward. The third and the fourth procedures also use all these data and are based on the GP model or on the ME model, respectively. The fifth procedure analyzes the same data with the MUSIC algorithm. As it is often difficult to define the number of incident wave fronts to be used with this method, we have always calculated the results for both one and two wave fronts. The position of the single wave front normal is indicated by an open triangle. In the same diagram we have plotted the results for two simultaneous wave fronts by two solid circles. Their positions again indicate the normal directions, and the size corresponds to the relative intensity of the respective wave front.

In the first example point, the plane wave analysis shows a downgoing wave at $\theta$ of $30^{\circ}$, propagating with $\phi$ of $45^{\circ}$ in the magnetic SW direction. The WDF analysis confirms that a high value of the polarization degree is well consistent with the plane wave hypothesis. The first WDF estimation using only the magnetic field data (DR-B) gives two exactly antiparallel peaks for both downgoing and upgoing waves. The addition of the electric field data allows us to confirm that the waves are downgoing: All the three parametric models in the first row of Figure 4 give a sharp concentration of wave energy near a single direction. This WDF peak is centered on the direction predicted with the plane wave approximation. The weak residual peak of upgoing waves obtained with the DR model is probably an artifact due to an insufficient resolution power of the data involving only one electric component. The results of the MUSIC algorithm are consistent with other methods. For a single wave front we obtain a direction very close to the previous plane wave estimate. This is also the rule for all following example points. Supposing two simultaneously propagating wave fronts, we obtain $\sim 75 \%$ of the wave energy in nearly the same direction as with the sin- gle wave front. The remaining energy is found not far from this direction, at an angular distance approximately corresponding to the width of the WDF peak obtained by other methods.

Point 2 is located in the most intense part of the ELF hiss emission, and in the frequency interval just above $f_{\mathrm{H}^{+}}$. The plane wave method gives a nearly field-aligned downward propagation at $\theta$ of $8^{\circ}$, but a lower polarization degree indicates the invalidity of the plane wave hypothesis. This well corresponds to the obtained WDFs. The result obtained with the magnetic components shows the same two-peaked shape in both diagrams. With addition of the electric component the downgoing peak is stronger and contains $\sim 80 \%$ of the total wave energy density. Comparing to the plane wave estimate, it deviates more from $\mathbf{B}_{0}$, with $\theta$ of around $30^{\circ}$, and with $\phi$ corresponding again to the $\mathrm{SW}$ direction. From the DR and GP models we have a large peak (a weak angular concentration of wave energy). The ME model gives a somewhat narrower peak near the same central direction. All the methods but the MUSIC algorithm give the second upgoing peak at higher $\theta$ and propagating again in the SW direction. This direction could be consistent with the hypothesis that this second peak originates by reflection of the downgoing waves at altitudes below the satellite orbit. However, the data could also be consistent with a single large peak of downgoing waves. This is indicated by the results of the MUSIC algorithm. With two incident wave fronts the ideal solution is found in two directions of downgoing waves with about equal intensities. The directions are near the WDF maxima obtained by other methods, and their angular distance roughly corresponds to the width of the WDF peak. However, a result which is very near to the simultaneous downgoing and upgoing propagation found by other methods can also be obtained with the MUSIC algorithm. This method gives here a great number of possible candidate directions (corresponding to local minima of the objective function: see the appendix for details); nevertheless, such a solution is not shown, as these other candidate directions have lower selection priorities (lower depths of the objective function minima). The uncertainty about the presence of the upgoing peak is probably again due to a lower resolution power using only one electric antenna. Point 3 is situated at the same time as the previous point, but in the higher-frequency interval of the hiss, where a higher polarization degree has been found. The downward and field-aligned propagation ( $\theta$ of $4^{\circ}$ ) is again detected by the plane wave analysis. Using all data available, the first two WDF models give a single peak of downgoing waves which is well concentrated around the plane wave estimate. With the ME model we obtain a slight shift of the WDF toward higher $\theta$. This difference is, however, not very high and is not of great importance for the overall wave propagation properties inside the hiss emission. The MUSIC algorithm again places the single wave front normal in the close vicinity of the previous plane wave estimate. For two wave fronts we obtain $\sim 75 \%$ of the intensity just next to this direction, and the rest is located on the west side at higher $\theta$, which is possibly consistent with the position of a weak "halo" in the WDF shape obtained with the DR model.

A more complex propagation is encountered in point 4 . It is located after the main part of the emission, and the plane wave methods recognize upward turning, NE directed wave vectors at $\theta$ of $150^{\circ}$, but the polarization degree is rather low. The WDFs we have obtained in this point show that the wave energy is concentrated around two main peaks. These peaks appear in both WDF diagrams obtained with the magnetic field compo- 
nents. With electric and magnetic data the upgoing peak and the downgoing peak are separated, similarly as in point 2 . The more intense one, with $\sim 80 \%$ of energy, is in the upward direction. Both peaks are at $\operatorname{similar} \theta$ which is higher than the plane wave estimate and slightly varies with the different WDF models. The important common result is that both peaks are in the NE direction, which supports the hypothesis that the upgoing peak is due to reflection of the downgoing waves at lower altitudes. The MUSIC algorithm is here perfectly consistent with other methods, and for two wave fronts the obtained directions and intensities are near the central directions and intensities of the two peaks modeled with Gaussian functions (GP). At higher frequencies, in point 5 , the plane wave methods give downgoing waves at $\theta$ of $50^{\circ}$, propagating again in the NE direction. According to a higher degree of polarization we would expect a similar concentration of wave energy density as in the higherfrequency band of the main part of the emission (point 3). The resulting WDFs show that this expectation is nearly appropriate A single peak of downgoing waves is found around the plane wave estimate. Its location is approximately the same as for the downgoing waves at lower frequencies in the previous point 4. The total energy density is, however, lower by $\sim 2$ orders of magnitude at higher frequencies because of much lower wave intensity. Small secondary peaks obtained with the DR and ME models have no counterpart in the results of other models. They can be probably interpreted as artifacts of the respective methods. The result of the MUSIC algorithm for a single wave front again coincides with the simple plane wave estimate, and for two wave fronts the procedure gives $100 \%$ of intensity in a direction near the peak obtained with the ME model, the second wave front being of zero intensity. In the band-limited part of the hiss emission, represented by the last point 6 , the plane wave analysis finds upward propagation. The waves are seen at higher $\theta$ of $\sim 130^{\circ}$, again in the NE direction. The WDF shapes obtained by the independent methods show a common feature: a strong peak of upgoing waves at $\theta$ higher than the plane wave estimate, but with a similar $\phi$ angle. Upward propagation with highly oblique wave vectors is also confirmed by the MUSIC procedure using a single wavefront. With two wave fronts, $\sim 85 \%$ of the intensity is found in the same direction, and the rest is placed in the direction which may very roughly correspond to a weak secondary peak in the WDF shape given by the DR model.

\section{Discussion}

In some cases, we have found that the assumption of the presence of a single plane wave is very far from reality (example points 2 and 4) and that the WDF or equivalent approach is necessary for a realistic description of wave propagation. The WDF analysis may be helpful even if the wave propagation can be well described by the model of a single plane wave (as in example point 1). This technique allows one to include the signais of the electric antennae, and independent recognition of upgoing and downgoing waves can be done. The WDF analysis also provides an appropriate tool to verify initial assumptions about a given plasma model by comparison of theoretical calculations of wave fields and measured field components. With a known theoretical dispersion relation the results of the WDF analysis can be easily used to estimate the distribution of wave energy over wavelengths.
All the WDF models but the DR model have some parameters which must be adjusted before each run. The inconvenience is that subjective adjustments may influence the final result. For the GP model we must choose the number of peaks. If this number is high, the optimization procedure finds no solution because the theoretical spectral matrix becomes insensitive to variations of different model parameters. We have therefore proceeded with a single peak, and when the peak was too wide (more than $45^{\circ}$ ), we have tried to find a solution for a model including two peaks, as in example points 2 and 4 . Similar rather subjective choice is connected with the ME model, where the number of model parameters must be adjusted. We have always tried to find solutions for all possible values of this number, and we have chosen a solution which exhibits acceptable stability and prediction results (see appendix for details). With the MUSIC algorithm we must choose a number of incident wave fronts. This task is well defined when the WDF is composed of distinct plane waves and the data are without noise: The number of wave fronts is equal to the number of nonzero eigenvalues of the spectral matrix. The problem is not so straightforward with real data, because the eigenvalues are not clearly clustered, even when the hypothesis of a single plane wave is assumed to be valid (as in example point 1). We have therefore proceeded with both one and two wave fronts. The results show that in the cases where the wave energy is concentrated near a single direction the procedure with two wave fronts gives also reasonable results, suppressing the intensity of the second wave front or finding one direction close to the other.

As the WDF is optimized to reach consistence between experimental and theoretical components of the spectral matrix, it is clear that the more signals of different antennae can be used, the better the quality of the WDF estimation is. In this paper, we have used the data of three magnetic and one electric antennae, which is the minimum configuration to recognize downgoing and upgoing waves. Some ambiguities, like these encountered in example point 2 would be probably better resolved with an increased number of antennae. The electric signals, however, induce several possible problems. First, the antenna is spinning, and we must assume an average antenna direction during the time interval of the spectral analysis. This may influence the results if the time of analysis is not much smaller than the spin period. To reduce this problem, we have always analyzed only one quarter of a 3-s data snapshot, and during this interval the antenna direction rotates by $\sim 45^{\circ}$. This angle cannot be further reduced because we must conserve a sufficient frequency resolution (we use a resolution of $16 \mathrm{~Hz}$ ) and a sufficient data averaging (we average 23 spectral matrix estimates with a time domain overlapping of $50 \%$ ). The second problem is connected with an unknown impedance between the electric antenna and the plasma medium. Again, this impedance can shift the phase of the electric signal or modify its strength, and the obtained WDF may be biased. We have observed that even small changes in the transfer function of the electric antenna can sometimes induce great changes of the resulting WDFs. Therefore we have tried to eliminate this problem by estimating the unknown impedance as a part of the WDF optimization procedure. These estimates give phase shifts between $-6^{\circ}$ and $-15^{\circ}$ and an amplitude between 70 and $100 \%$ of the original value in the plasma medium. Because of these uncertainties connected with the electric antenna we always compare the result with the WDF which is estimated with the magnetic field data only. The results are consistent if all structures of the WDF shape do not much differ. We can see 
that this is the case of the results in all the points $1-6$, and this suggests that the unknown biases are well corrected.

\section{Conclusions}

We have analyzed an example case of electromagnetic ELF hiss event observed at low altitudes by the Freja satellite. We have compared the results of both plane wave techniques and several WDF analysis methods. For higly polarized waves all the WDF methods give similar results as obtained by the plane wave approach. When the polarization degree is low, downgoing and reflected upgoing waves are simultaneously observed, or a large distribution of wave energy with respect to wave-normal directions is found. In this case the WDF techniques become necessary for a realistic description of the wave propagation. The WDF analysis also confirms that experimental polarization properties well correspond to their predictions from the cold plasma theory. Comparing the results obtained by four different methods, we cannot say that they are absolutely equal, because of different constraints imposed by the WDF models. However, these results allow a common interpretation. The observed differences are probably due to ambiguities induced by the data and/or by the inversion methods themselves. These differences also demonstrate the limitations of the WDF analysis with our particular data set.

\section{Appendix: WDF Analysis Methods}

The procedure of the WDF analysis can be divided into two separate tasks: (1) the direct problem and (2) the inverse problem. The direct problem consists of the theoretical prediction of experimentally accessible wave data for a given WDF shape. All methods developed up to now are restricted to the linear theory of homogeneous media, and the predicted data are generally represented by the spectral matrices of several electric or magnetic antennae at given frequencies. The WDF direct problem was first resolved by Storey and Lefeuvre $[1979,1980]$, who used a cold plasma approximation to obtain algebraic expressions for the components of the spectral matrix. A kinetic extension of this method is presented by Santolik [1995] and Santolik and Parrot [1996]. The latter numerical procedure involving a simplified cold plasma approach has been used in the present paper. In this procedure the theoretical components $S_{i j}$ of the spectral matrix at a given frequency $\omega$ are calculated by a discrete version of the original integral equation of Storey and Lefeuvre,

$$
S_{i j}=\sum_{m} \sum_{\ell=1}^{L_{m}} a_{m i j}\left(\theta_{m \ell}, \phi_{m \ell}\right) G_{m}\left(\theta_{m \ell}, \phi_{m \ell}\right) \sigma_{m \ell}
$$

where $i, j=1, \ldots, N, N$ is the number of antennae, $\ell$ is the index specifying one of the $L_{m}$ predefined wave vector directions (see Figure 1) for a wave mode $m, \sigma_{m \ell}$ is an elementary solid angle, $a_{m i j}$ are the integration kernels calculated usin. the theoretical properties of a given wave mode, and $G_{m}$ is the WDF of the mode $m$.

The WDF inverse problem is the estimation of a WDF shape which is consistent with measured wave data. The inverse procedure must be obviously based on a method resolving the direct problem. All the existing inversion procedures use iterative algorithms which search for WDFs giving theoretical spectral matrices consistent with the experimental ones. The procedures also involve a regularization of the underdetermined inverse problem. The regularization could be done either by a constrained optimization of a regularization parameter, or it could be directly contained in a shape of a parametric WDF model [Lefeuvre and Delannoy, 1979]. In the latter case, the WDF is defined by $M$ parameters, which together form an $M$ dimensional parameter vector $\mathbf{P}$. This vector is estimated by a nonlinear maximum-likelihood procedure which minimizes the $\chi^{2}$ value,

$\chi^{2}=\sum_{j \geq i}\left(\frac{\Re \hat{S}_{i j}-\Re S_{i j}(\mathbf{P})}{\Re\left(\delta \hat{S}_{i j}\right)}\right)^{2}+\left(\frac{\Im \hat{S}_{i j}-\Im S_{i j}(\mathbf{P})}{\Im\left(\delta \hat{S}_{i j}\right)}\right)^{2}$

where $\hat{S}_{i j}$ are the measured spectral matrix components, $\delta \hat{S}_{i j}$ are the corresponding experimental errors (statistical standard deviations of the spectral analysis), and $S_{i j}(\mathbf{P})$ are the theoretical predictions from A1 for a parametrized WDF. Note that $\hat{S}_{i j}$ and $S_{i j}(\mathbf{P})$ are Hermitian matrices. In the present paper we use three different parametric models based on this approach: the maximum entropy (ME) model, the model of Gaussian peaks (GP), and the model of discrete regions (DR). Besides these "least squares" methods we use also an independent analysis procedure based on the interpretation of the spectral matrix eigenstructure. In the following, these four WDF estimation methods are described in more detail.

\section{A1. Maximum Entropy Model}

Lefeuvre and Delannoy [1979] developed a computer program MAXENTWDF based on the parametric ME model. A detailed documentation is given by Delannoy and Lefeuvre [1986]. The WDF model reads

$$
G_{m}(\theta, \phi)=\exp \left(-1+\sum_{k=1}^{M} \lambda_{k} \Pi_{k}(\theta, \phi)\right),
$$

where $\lambda_{k}$ are the model parameters. The number $M$ of parameters must be chosen to have $M \leq N^{2}$. The functions $\Pi_{k}(\theta, \phi)$ are calculated as a linear combination of integration kernels $a_{m i j}$ to remove the mutual dependence of model parameters. The parameters are optimized by an iterative least squares procedure to minimize a transformed version of $A 2$. The reliability of the resulting WDF can be judged using several parameters. The prediction parameter $P_{r}$ defines the global disagreement between the measured and modeled spectral matrices. Acceptable values are $P_{r} \leq 1.5$ [Lefeuvre et al., 1992]. The stability parameter $S_{t}$ describes the stability of the solution compared to the data variance. Lefeuvre et al. [1992] found stable solutions for $S_{t}$ values up to 5 . In the present paper we use four antennae $\left(N^{2}=16\right)$ and $M$ between 10 and 13. We obtain $P_{r}$ between 1.1 and 1.5 and $S_{t}$ between 1.2 and 4.2 .

\section{A2. Model of Gaussian Peaks}

Concentration of wave energy near one or several wavenormal directions is often observed [e.g., Lefeuvre et al., 1986, 1992; Parrot and Lefeuvre, 1986]. In such a case, reasonable information about the WDF can be reduced to a set of the peak directions and accompanied by the respective energy densities and measures of wave energy concentration near the respective directions (peak widths). We model the WDF as a superposition of $K$ peaks [Santolik, 1995], 


$$
G_{m}(\theta, \phi)=\sum_{k=1}^{K} G_{m_{k}}(\theta, \phi) .
$$

Each peak is represented by a Gaussian function in 2-D space of wave-normal directions (or, equivalently, on the surface of a unit sphere),

$$
G_{m_{k}}(\theta, \phi)=\frac{\rho_{k}}{\pi \Delta_{k}^{2}} \exp \left(-\frac{D_{k}^{2}}{\Delta_{k}^{2}}\right)
$$

where $\rho_{k}$ is the peak energy and $\Delta_{k}$ is the peak width, assuming the angular deviation $D_{k}$ measured between a direction $(\theta, \phi)$ and the central direction $\left(\theta_{k}, \phi_{k}\right)$ of the $k$ th peak,

$$
D_{k}^{2}=2\left[1-\cos \theta_{k} \cos \theta-\sin \theta_{k} \sin \theta \cos \left(\phi_{k}-\phi\right)\right] .
$$

The model has $4 K$ free parameters $\left(\theta_{k}, \phi_{k}, \rho_{k}, \Delta_{k}, k=1, \ldots\right.$, $K$ ) which are optimized using the nonlinear LevenbergMarquardt procedure [Press et al., 1992] to obtain minimum $\chi^{2}$ value (A2). The number of peaks must be chosen to have $4 K<N^{2}$, and good initial estimates of model parameters are necessary to reach the convergence of the iterative optimization procedure. To overcome this necessity, the optimization is run repeatedly with randomly initiated parameters and a limited number of iterations; the result where the convergence has been achieved and which has the lowest $\chi^{2}$ is chosen.

\section{A3. Model of Discrete Regions}

The third model is simple and rather general. The space of wave-normal directions (equivalent to the $2-\mathrm{D}$ surface of the unit sphere) is divided into $J$ discrete regions. A coverage similar to the layout of snow bricks in an igloo (see Figure 2 of Santolik and Parrot [1996]) is generally a good choice because we obtain about constant solid angles (2-D surfaces) corresponding to the respective regions. The WDF is supposed to be equal to a constant $G_{m}\left(\theta_{j}, \phi_{j}\right)$ in each region surrounding a central direction $\left(\theta_{j}, \phi_{j}\right)$. These $K$ WDF values represent directly the optimized model parameters. The WDF cannot obviously be negative, and a simple linear inversion procedure is therefore useless. The optimization of A2 is done with the iterative Levenberg-Marquardt procedure [Press et al., 1992]. The procedure is modified to implement the constraint of positive or zero parameter values by the method of projections onto convex sets [Youla and Webb, 1982]. In this case, the optimization gives useful results even if the number of model parameters is much greater than the number of spectral matrix elements, $J \gg N^{2}$. For the WDF reconstructions presented in this paper we use $J$ $=412$ to cover the full $4 \pi$ solid angle. This is possible because an inherent regularization of the underdetermined problem is contained in the optimization procedure. As shown by Biemond et al. [1990], a similar image restoration problem is sensitive to the final number of iterations, i.e., to the criterion of convergence. To obtain acceptable results, we stop the iterations if none of the parameters (WDF values) change by more than $1 \%$ in the last three successful iterations.

\section{A4. A WDF Implementation of the Multiple Signal Classification (MUSIC) Algorithm}

This method calculates the wave-normal directions and intensities of multiple plane waves using the multiple signal classification (MUSIC) algorithm of Schmidt [1981, 1986]. An im- plementation of this algorithm for the WDF analysis has been done by Storey [1993]. It can accommodate any number $K$ of plane waves less than the number $N$ of measured field components. This model of the WDF is deemed applicable if one or more eigenvalues of the spectral matrix are very much smaller than the largest one. The user must decide the number $K$ of wave fronts to be used in modeling the WDF. If the model were exactly valid and the data were free from noise, the right choice would be the number of nonzero eigenvalues. In a real case, however, it may be hard to tell whether or not a certain eigenvalue differs significantly from zero. The MUSIC algorithm works in a different way compared to the above-described maximum-likelihood methods. From the eigenstructure of the spectral matrix and from the integration kernels $a_{m i j}$, it defines an "objective function" which depends on just two variables, the angles $\theta$ and $\phi$. It has the property that if the data are noiseless, it vanishes in the directions of the $K$ wave normals. Thus the determination of the wave-normal directions is reduced to locating the minima of the objective function on the unit sphere. It often happens that the procedure finds more wave-normal directions than are required, and then the user must decide which are the most probable ones. In the normal way these should be the $K$ wave fronts for which the values of the objective function are smallest. As soon as these most probable directions are known, the amplitudes can be obtained by a simple calculation.

Acknowledgments. Many thanks are due to B. Holback (IRF-U), the PI of the F4 experiment, for the use of his data and for helpful discussions about the data handling. Data are available in France thanks to H. de Feraudy (CETP-UVSO), who is Co-I of F4. Important software has been used for the data analysis in this paper: ISDAT (Interactive Science Data Analysis Tool), developed by A. Lundgren (IRF-U), and SWAN (Software for Waveform Analysis), developed by D. Lagoutte (LPCE). We thank L.R.O. Storey, who has provided us with his computer program MUSIC. This work was supported by the French-Czech program Barrande $98039 / 98055$ and by the international program of scientific cooperation (PICS) 469 with the joint Czech Grant Agency grant 205/98/0691. O. Santolik acknowledges the support of the Czech Grant Agency grant 205/99/1712.

Michel Blanc thanks the three referees for their assistance in evaluating this paper.

\section{References}

Biemond, J., R.L. Lagendijk, and R.M. Mersereau, Iterative methods for image deblurring, Proc. IEEE, 78, 856-883, 1990.

Delannoy, C., and F. Lefeuvre, MAXENTWDF: A computer program for the maximum entropy estimation of a wave distribution function, Comput. Phys. Commun., 40, 389-419, 1986.

FREJA magnetic field experiment team, Magnetic field experiment on the Freja satellite, Space Sci. Rev., 70, 465-482, 1994.

Gurnett, D. A., and T. B. Burns, The low-frequency cutoff of ELF emissions, J. Geophys. Res., 73, 7437-7445, 1968.

Holback, B., S.-E. Jansson, L. Ahlen, G. Lundgren, L. Lyngdal, S. Powell, and A. Meyer, The FREJA wave and plasma density experiment, Space Sci. Rev., 70, 577-592, 1994.

Lefeuvre, F., and C. Delannoy, Analysis of random electromagnetic wave field by a maximum entropy method, Ann. Telecommun., 34, 204-213, 1979.

Lefeuvre, F., and R.A. Helliwell, Characterization of the sources of VLF hiss and chorus observed on GEOS 1, J. Geophys. Res., 90, 6419-6438, 1985.

Lefeuvre, F. Y. Marouan, M. Parrot, and J.L. Rauch, Rapid determination of the sense of polarization and propagation for random electromagnetic wave fields: Application to GEOS1 and AUREOL3 data, Ann. Geophys. Ser. A, 4, 457-468, 1986.

Lefeuvre, F., J. L. Rauch, D. Lagoutte, J. J. Berthelier, and J. C. Cerisier, Propagation characteristics of dayside low-altitude hiss: Case studies, J. Geophys. Res., 97, 601-620, 1992. 
McPherron, R. L., C. T. Russel, and P. J. Coleman Jr., Fluctuating magnetic fields in the magnetosphere, 2, ULF waves, Space Sci. Rev., 13, 411-454, 1972.

Means, J. D., Use of the three-dimensional covariance matrix in analyzing the polarization properties of plane waves, J. Geophys. Res. $77,5551-5559,1972$.

Parrot, M., and F. Lefeuvre, Statistical study of the propagation characteristics of ELF hiss observed on GEOS-1, outside and inside the plasmasphere, Ann. Geophys. Ser. A, 4, 363-384, 1986.

Press, W. H., B. P. Flannery, S. A. Teukolsky, and W. T. Vetterling Numerical Recipes, Cambridge Univ. Press, New York, 1992.

Samson, J. C., and J. V. Olson, Some comments on the descriptions of the polarisation states of waves, Geophys. J. R. Astron. Soc., 61 $115-129,1980$

Santolík, O., Etude de la fonction de distribution des ondes dans un plasma chaud (in French), Ph.D. thesis, Univ. d'Orléans, Orléans, France, 1995.

Santolik, O., and M. Parrot, The wave distribution function in a hot magnetospheric plasma: The direct problem, J. Geophys. Res., 101, $10,639-10,651,1996$.

Santolík, O., and M. Parrot, Propagation analysis of electromagnetic waves between the helium and proton gyrofrequencies in the lowaltitude auroral zone, J. Geophys. Res., 103, 20,469-20,480, 1998.

Santolík, O., and M. Parrot, Case studies on the wave propagation and polarization of ELF emissions observed by Freja around the local proton gyrofrequency, J. Geophys. Res., 104, 2459-2475, 1999.

Schmidt, R. O., A signal subspace approach to multiple emitter location and spectral estimation, Ph.D. thesis, 201 pp., Stanford Univ., Stanford, Calif., Nov. 1981.

Schmidt, R. O., Multiple emitter location and signal parameter estimation, IEEE Trans. Antennas Propag., AP-34, 276-280, 1986.
Stix, T. H., Waves in Plasmas, Am. Inst. of Phys., New York, 1992.

Storey, L. R. O., Direction-finding for multiple whistler-mode waves in space (abstract), Eos Trans. AGU, 74(16), Spring Meet. Suppl., S271, 1993

Storey, L. R. O., and F. Lefeuvre, Theory for the interpretation of measurements of a random electromagnetic wave field in space, Space Res., 14, 381-386, 1974.

Storey, L. R. O., and F. Lefeuvre, The analysis of 6-component measurement of a random electromagnetic wave field in a magnetoplasma, 1 , The direct problem, Geophys. J. R. Astron. Soc., 56, 255-270, 1979.

Storey, L. R. O., and F. Lefeuvre, The analysis of 6-component measurement of a random electromagnetic wave field in a magnetoplasma, 2 , The integration kernels, Geophys. J. R. Astron. Soc., 62, 173-194, 1980.

Storey, L. R. O., F. Lefeuvre, M. Parrot, L. Cairó, and R.R. Anderson, Initial survey of the wave distribution functions for plasmaspheric hiss observed by ISEE 1, J. Geophys. Res., 96, 19,469-19,489, 1991.

Youla, D.C., and H. Webb, Image restoration by the method of convex projections, part 1, Theory, IEEE Trans. Medical Imaging, MI-1, $81-94,1982$.

M. Parrot, Laboratoire de Physique et Chimie de l'Environnement, CNRS, 3A, Avenue de la Recherche Scientifique, 45071 Orléans cedex 02, France. (mparrot@ cnrs-orleans.fr)

O. Santolík, Faculty of Mathematics and Physics, Charles University, V Holešovičkách 2, CZ-18000 Praha 8, Czech Republic. (ondrej.santolik@mff.cuni.cz)

(Received December 9, 1998; revised December 13, 1999; accepted February 8, 2000.) 